\section{(2) OPEN ACCESS}

\title{
Lactobacillus gallinarum modulates the gut microbiota and produces anti-cancer metabolites to protect against colorectal tumourigenesis
}

\author{
Naoki Sugimura, ${ }^{1,2}$ Qing Li, ${ }^{1,3}$ Eagle Siu Hong Chu, ${ }^{1}$ Harry Cheuk Hay Lau (D) ,' \\ Winnie Fong, ${ }^{1}$ Weixin Liu, ${ }^{1}$ Cong Liang, ${ }^{4}$ Geicho Nakatsu, ${ }^{5}$ Anthony Chin Yang Su, \\ Olabisi Oluwabukola Coker, ${ }^{1}$ William Ka Kei Wu (D) , ${ }^{3}$ Francis Ka Leung Chan (D) , \\ Jun Yu (iD) ${ }^{1}$
}

Additional supplemental material is published online only. To view, please visit the journal online (http://dx.doi.org/ 10.1136/gutjnl-2020-323951).

For numbered affiliations see end of article.

\section{Correspondence to} Dr Jun Yu, Medicine and Therapeutics, The Chinese University of Hong Kong, Hong Kong, Hong Kong: junyu@cuhk.edu.hk Professor Francis Ka Leung Chan; fklchan@cuhk.edu.hk

NS and QL are joint first authors.

Received 22 December 2020 Accepted 7 December 2021
Check for updates

(c) Author(s) (or their employer(s)) 2021. Re-use permitted under CC BY-NC. No commercial re-use. See rights and permissions. Published by BMJ.

To cite: Sugimura $\mathrm{N}$, Li Q Chu ESH, et al. Gut Epub ahead of print: [please include Day Month Year]. doi:10.1136/

gutinl-2020-323951

\section{ABSTRACT \\ Objective Using faecal shotgun metagenomic} sequencing, we identified the depletion of Lactobacillus gallinarum in patients with colorectal cancer (CRC). We aimed to determine the potential antitumourigenic role of $L$. gallinarum in colorectal tumourigenesis.

Design The tumor-suppressive effect of $L$. gallinarum was assessed in murine models of CRC. CRC cell lines and organoids derived from patients with CRC were cultured with L. gallinarum or Escherichia coli MG1655 culture-supernatant to evaluate cell proliferation, apoptosis and cell cycle distribution. Gut microbiota was assessed by $16 \mathrm{~S}$ ribosomal DNA sequencing. Antitumour molecule produced from L. gallinarum was identified by liquid chromatography mass spectrometry (LC-MS/MS) and targeted mass spectrometry.

Results L. gallinarum significantly reduced intestinal tumour number and size compared with E. coli MG1655 and phosphate-buffered saline in both male and female murine intestinal tumourigenesis models. Faecal microbial profiling revealed enrichment of probiotics and depletion of pathogenic bacteria in L. gallinarumtreated mice. Culturing CRC cells with L. gallinarum culture-supernatant $(5 \%, 10 \%$ and $20 \%$ ) concentrationdependently suppressed cell proliferation and colony formation. L. gallinarum culture-supernatant significantly promoted apoptosis in CRC cells and patient-derived CRC organoids, but not in normal colon epithelial cells. Only L. gallinarum culture-supernatant with fraction size $<3 \mathrm{kD}$ a suppressed proliferation in CRC cells. Using LC-MS/MS, enrichments of indole-3-lactic acid (ILA) was identified in both L. gallinarum culture-supernatant and the gut of $L$. gallinarum-treated mice. ILA displayed anti-CRC growth in vitro and inhibited intestinal

tumourigenesis in vivo.

Conclusion L. gallinarum protects against intestinal tumourigenesis by producing protective metabolites that can promote apoptosis of CRC cells.

\section{INTRODUCTION}

Colorectal cancer (CRC) is the third most commonly diagnosed malignancy and the second leading cause of cancer death in the world. ${ }^{1}$ There are many risk factors associated with CRC carcinogenesis including genetic alterations, lifestyle and

\section{Significance of this study}

What is already known on this subject?

- Lactobacillus gallinarum is one of the most depleted probiotic species in the stool of patients with colorectal cancer (CRC).

What are the new findings?

- Administration of L. gallinarum inhibited colorectal tumourigenesis in $\mathrm{Apc}^{\mathrm{Min} /+}$ mice and in azoxymethane/dextran sulfate sodiumtreated mice.

- L. gallinarum increased abundances of gut probiotics and depleted potential gut pathogens.

- L. gallinarum culture-supernatant suppressed cell proliferation, and induced apoptosis in CRC cell lines and in organoids derived from patients with CRC.

- Secreted non-protein molecule(s) with a molecular weight $<3 \mathrm{kDa}$ from $L$. gallinarum mediated the anti-CRC effect.

- Indole-3-lactic acid, a small molecule with known anti-inflammatory property, was identified as the most enriched metabolite produced by L. gallinarum .

How might it impact on clinical practice in the foreseeable future?

- The probiotic L. gallinarum is a potential prophylactic for preventing CRC in humans.

environmental factors. ${ }^{2}$ Over the last decade, gut microbiota has been shown to play a key role in CRC development. Certain probiotic bacteria such as Streptococcus thermophilus and Lactobacillus rhamnosus have demonstrated anticarcinogenic properties. $^{34}$

Most Lactobacillus species are classified as lactic acid bacteria (LAB). LAB are generally found in fermented food, such as decomposing plants and milk products, and they are widely accepted to be used as probiotics for humans. ${ }^{5}$ The beneficial effects of LAB for diseases have been reported extensively ${ }^{67}$ and preclinical studies have shown its abilities to reduce chronic inflammation associated with 
cancer development. ${ }^{89}$ Using shotgun metagenomic sequencing, we identified a probiotic species Lactobacillus gallinarum being significantly depleted in the stool of patients with CRC, ${ }^{10}$ suggesting that it might play a role in suppressing CRC. In this study, we showed that L. gallinarum could abrogate colorectal tumourigenesis in mouse models, human CRC-derived organoids and CRC cell lines through promoting apoptosis. This tumour-suppressing effect was attributed to indole-3-lactic acid (ILA), a metabolite generated by L. gallinarum .

\section{MATERIALS AND METHODS Animal experiments}

Male and female $A p c^{\mathrm{Min} /+}$ C57B/6 mice, which can faithfully recapitulate the human familial adenomatous polyposis, was used as a mouse model of spontaneous CRC. ${ }^{11} A p c^{\mathrm{Min} /+}$ mice at 5-6 weeks old were divided into three groups with or without CH-223191 treatment: (1) phosphate-buffered saline (PBS); (2) Escherichia coli MG1655 and (3) L. gallinarum. Lactobacillus casei was further used as a LAB control. L. gallinarium was cultured in MRS broth (Difco Laboratories, Detroit, Minnesota, USA) and L. casei and E. coli MG1655 were cultured in brain heart infusion (BHI) broth. After 1 day, each of the bacteria was collected and resuspended in PBS and then gavaged to mice $\left(1 \times 10^{8}\right.$ colony-forming unit (CFU) $/ 100 \mu \mathrm{L}$ PBS per mouse). Male and female mice were gavaged once daily for 8 and 12 weeks, respectively, for the development of neoplastic lesions. Body weight and stool were examined weekly.

The azoxymethane (AOM)/dextran sulfate sodium (DSS) model, which can mimic human colitis-associated carcinoma (CAC), was also established. Male and female C57BL/6 mice at 6 weeks old were intraperitoneally injected with a single dose of $10 \mathrm{mg} / \mathrm{kg}$ AOM (Merck, Darmstadt, Germany), followed by 2\% DSS (MP Biomedicals, Solon, Ohio, USA) administration for 1 week. AOM/DSS-induced CRC mice were gavaged with $L$. gallinarium and E. coli MG1655 suspension following the same schedule.

Mouse colonoscopy (Karl Storz Endoskope, Tuttlingen, Germany) was performed prior to sacrifice. The colonoscope was inserted into anus and advanced proximally under direct visualisation, facilitated by air insufflation, and representative pictures of the colon tumour from each group were recorded. After neoplastic lesions developed, mice were anaesthetised and sacrificed. Small intestines and colons of mice were longitudinally opened and rinsed with PBS. Total number of tumours in small intestine and colon were recorded. Size of each tumour was measured using previous published formula. ${ }^{12}$

\section{S rDNA gene sequence analysis}

Raw de-multiplexed FASTQ files were preprocessed in Mothur. ${ }^{13}$ Contigs were created using Needleman-Wunsch alignment algorithm with default parameters, ${ }^{14}$ and aligned against the SILVA database (V.123) using NAST algorithm. ${ }^{15} 16$ We removed any contigs with homopolymers of $>8$ nucleotides and retained all that mapped within the identical coordinates. Any sequence pairs with mismatch difference of $\leq 2$ were preclustered to reduce amplicon sequencing noises. Chimeric sequences were culled using de novo UChime. ${ }^{17}$ Postquality controlled sequences were classified using Greengenes 16S rRNA database (V.13.8). We discarded any sequences of eukaryotic, archaea, mitochondrial, chloroplastic or unknown origins before binning them into operational taxonomic units (OTUs) at 97\% identity threshold. The lowest taxonomic annotation for an OTU was defined as having a consensus assignment score of $\geq 80$. Sequence count table was rarefied to the smallest number of reads per sample (ie, 44845 reads) to reduce the effects of variable sequencing depths on downstream analyses. The significance of Alpha diversity was assessed by Wilcoxon rank-sum test. Beta diversity was visualised by principal coordinate analysis. P value $<0.05$ was considered statistically significant. Differential abundance analysis was performed with one-way analysis of variance (ANOVA). Average fold change for each OTU and heatmap was computed in the R Project for Statistical Computing. ${ }^{18}$ The $\mathrm{p}$ values obtained were adjusted for multiple comparisons by the false discovery rate method. The corresponding $\mathrm{q}<0.05$ was considered statistically significant.

\section{Bacterial strains and culture conditions}

L. gallinarum (ATCC 33199) and L. casei (ATCC BAA-2843) were purchased from American Type Culture Collection (ATCC; Manassas, Virginia, USA). E. coli strain MG1655 (ATCC 700926), a non-pathogenic human commensal intestinal bacterium, was included as a negative control. ${ }^{19}$ They were cultured in MRS broth or $\mathrm{BHI}$ broth as appropriate at $37^{\circ} \mathrm{C}$ under aerobic condition.

\section{CRC patient-derived organoid culture}

CRC organoids derived from 2 patients (74 and 816) were obtained from Princess Margaret Living Biobank (Toronto, Ontario, Canada), and embedded into Matrigel (Corning, Corning, New York, USA). Culture medium was changed every 2 days. After 5 days of culture, Matrigel was removed to expose organoids by mechanical stress and/or TrypLE digestion (SigmaAldrich). Organoids were then collected for further experiments. The detailed information about these two patients with CRC is listed in online supplemental table 1.

\section{Cell viability assay}

Cell viability was measured by 3-(4,5-dimethylthiazoly-2-yl )-2,5-diphenyltetrazolium bromide (MTT) (Sigma-Aldrich) assay. Cells were seeded on 96 -well plates at $1.0 \times 10^{3}$ cells per well and incubated for 24 hours before treatment. Cells were cultured in Dulbecco's Modified Eagle's Medium with addition of bacterial supernatants at different concentrations (5\%,10\% or $20 \%$ ) or the same amount of ILA (Sigma-Aldrich) comparable to that of $20 \%$ L. gallinarum culture supernatant (LGCS). Cell proliferation was measured by MTT assay for five consecutive days. The amount of MTT formazan product was determined by measuring absorbance at a wavelength of $570 \mathrm{~nm}\left(\mathrm{OD}_{570}\right)$ with a microplate reader (Multiskan GO Microplate Spectrophotometer, Thermo Scientific, Vantaa, Finland). For pharmacological inhibition of aryl hydrocarbon receptor (AhR), CH-223191 $(100 \mathrm{nM})$ was added 12 hours before the treatment with bacteria culture supernatant.

\section{Apoptosis assay and cell cycle analysis}

Cells were plated on 6-well plates 24 hours prior to treatment, and cultured in medium containing 10\% LGCS, E. coli culture supernatant (ECCS) or BHI. CRC patient-derived organoids were cultured on 6-well plates, and 10\% LGCS, ECCS or BHI was added to Matrigel and growth medium. After 5 days of treatment, cells and organoids were digested in $0.25 \%$ trypsinEDTA (Gibco-Invitrogen, Grand Island, New York, USA) and TrypLE, respectively. For apoptosis assay, the proportion of apoptotic cells was evaluated by dual staining with Annexin $\mathrm{V}-\mathrm{PE}$ and 7-aminoactinomycin D (7-AAD) (BD Pharmingen, San Jose, California, USA). Combination of Annexin V-PE and 


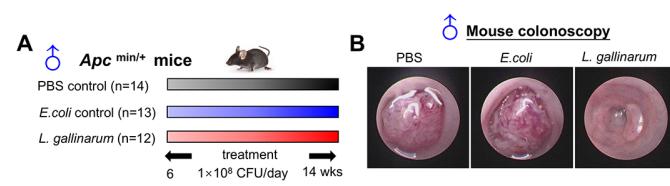

C
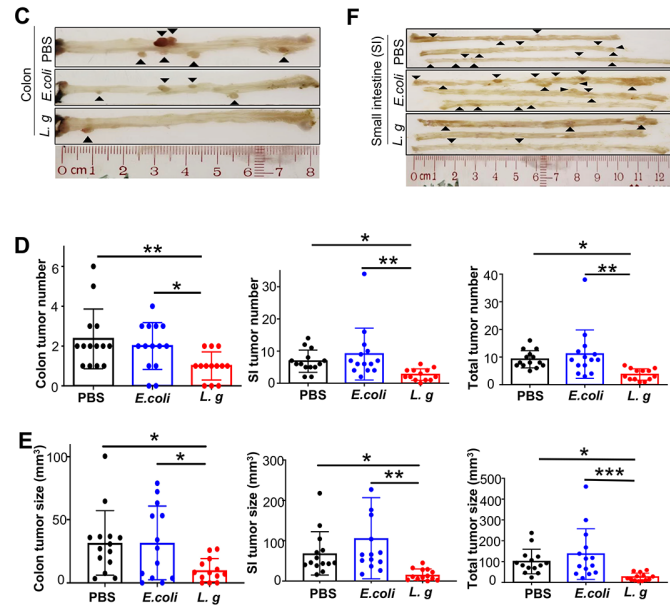
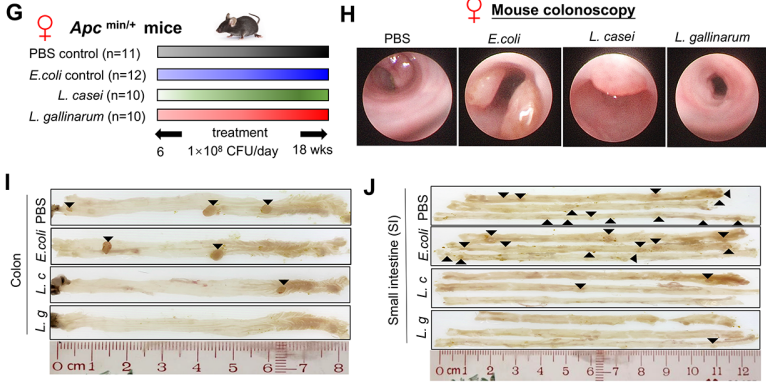

K
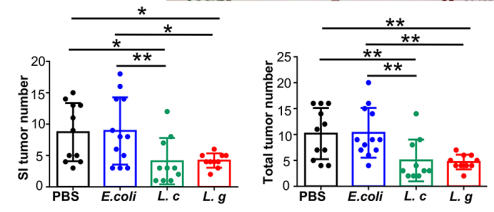

L

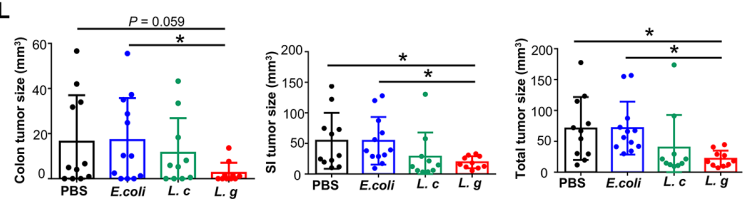

Figure 1 L. gallinarum protects against intestinal tumourigenesis in $A p C^{\mathrm{Min} /+}$ mice. (A) Schematic diagram showing the experimental design, timeline of male $A p \mathrm{CM}^{\mathrm{Mn} /+}$ mouse model. (B) Representative images of colon tumour from male $A p \mathrm{C}^{\mathrm{Min} /+}$ mouse model. Colonoscopy confirmed that colon tumour size in L. gallinarum group was visually smaller than tumours in Escherichia coli MG1655 or PBS control groups. In both E. coli MG1655 and PBS control groups, colonoscope could not pass through the tumours. (C) Representative macroscopic images of colons from male $\mathrm{ApC}^{\mathrm{Min} /+}$ mouse model. (D) Colon, small intestinal and total tumour number (colon+small intestinal) in male $A p c^{\text {Min/+ }}$ mice under different treatments. (E) Colon, small intestinal and total tumour size (colon+small intestinal) in male $A p C^{\mathrm{Min} /+}$ mice under different treatments. (F) Representative macroscopic images of small intestines from male $A p c^{\mathrm{Min} /+}$ mouse model. (G) Schematic diagram showing the experimental design, timeline of female $A p c^{\mathrm{Min} /+}$ mouse model. (H) Representative colonoscopic images of colon tumour from female $A p c^{\mathrm{Min} /+}$ mouse model. (I) Representative macroscopic images of colons from female $A p c^{\mathrm{Min} /+}$ mouse model. (J) Representative macroscopic images of small intestines from female $A p \mathrm{C}^{\mathrm{Min} / \mathrm{+}}$ mouse model. (K) Colon, small intestinal and total tumour number (colon+small intestinal) in female $\mathrm{Apc}^{\mathrm{Min} /+}$ mice under different treatments. (L) Colon, small intestinal and total tumour size (colon+small intestinal) in female $\mathrm{ApC}^{\mathrm{Min} /+}$ mice under different treatments. Each black triangle indicates one tumour location. P values are calculated by one-way analysis of variance. ${ }^{*} \mathrm{P}<0.05$, ${ }^{* *} \mathrm{p}<0.01$, ${ }^{* *} \mathrm{p}<0.001$. L. c, Lactobacillus casei; L. g, Lactobacillus gallinarum; PBS, phosphate-buffered saline; Sl, small intestine.

7-AAD staining distinguished early apoptotic cells (Annexin $\mathrm{V}^{+}$, 7-AAD ${ }^{-}$) and late apoptotic cells (Annexin $\mathrm{V}^{+}, 7-\mathrm{AAD}^{+}$). For cell cycle analysis, cells treated with 10\% LGCS, ECCS or BHI for 1 day were fixed and stained with $50 \mu \mathrm{g} / \mathrm{mL}$ propidium iodide (BD Pharmingen). Cell cycle of all stained cells were analysed using FASAria cell sorter (BD Biosciences, San Jose, California, USA).

\section{Liquid chromatography mass spectrometry (LC-MS/MS)- tandem mass spectrometry analysis for bacteria culture supernatant}

To investigate anti-CRC metabolites in LGCS, $100 \mu \mathrm{L}$ of each sample (LGCS, ECCS, BHI) was added to $400 \mu \mathrm{L}$ extraction solution containing internal standard (L-2-chlorophenylalanine, $2 \mu \mathrm{g} / \mathrm{mL}$ ). Following centrifugation, $75 \mu \mathrm{L}$ of supernatant was transferred to a fresh glass vial for liquid chromatography mass spectrometry (LC-MS/MS) analysis. Ultra-high performance liquid chromatography (UHPLC) separation was carried out using a 1290 Infinity series UHPLC System (Agilent Technologies, Palo Alto, California, USA), equipped with a UPLC BEH Amide column. The analysis was carried with elution gradient as follows: $0-0.5 \mathrm{~min},>95 \% \mathrm{~B}$; $0.5-7.0 \mathrm{~min}, 95 \%-65 \% \mathrm{~B}$; 7.0-8.0 min, 65\%-40\% B; 8.0-9.0 min, <40\% B; 9.0-9.1 min, 40\%-95\% B; 9.1-12.0 min, 95\% B. The TripleTOF 6600 mass spectrometry (AB Sciex, Foster city, California, USA) was used to acquire tandem mass spectrometry (MS/MS) spectra on an information-dependent basis during LC-MS/MS experiment. The processing of LC-MS/MS raw data was achieved using R package XCMS (V.3.2). Peak annotation was processed by CAMERA package implemented in the $\mathrm{R}$ language. In-house MS2 database, Human Metabolome Database (HMDB, www. hmdb.ca), and METLIN metabolite database (metlin.scripps. edu) were used for a more reliable metabolite identification. Significantly enriched metabolites were identified by a two-tailed Mann-Whitney U test. Compared with BHI or ECCS, specific metabolite enriched in LGCS with adjusted $\mathrm{p}$ value $<0.05$ was considered statistically significant.

\section{Statistical analysis}

Values are expressed as mean $\pm \mathrm{SD}$ for both in vivo and in vitro experiments. Comparisons between two groups were performed using a two-sided Student's t-test. ANOVA was used to compare differences among multiple groups, and post hoc analysis was performed by Tukey's multiple comparisons test. P value $<0.05$ indicates statistical significance.

Additional methods are provided in the online supplemental material 1.

\section{RESULTS}

\section{L. gallinarum protects against intestinal tumourigenesis in Apc $^{\mathrm{Min} /+}$ mice}

To investigate the effect of $L$. gallinarum on colorectal tumourigenesis, we first used male $A p c^{\mathrm{Min} /+}$ mice. We gavaged the mice with L. gallinarum $\left(1.0 \times 10^{8} \mathrm{CFUs}\right.$ per mouse), a non-tumourigenic 


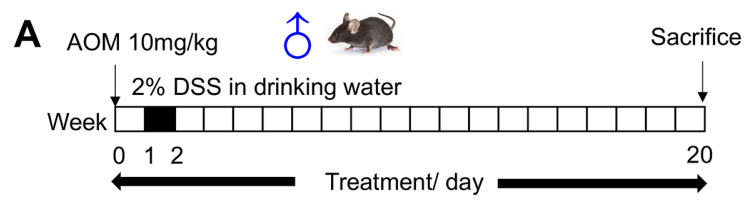

B

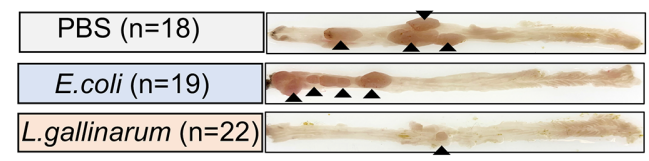

C
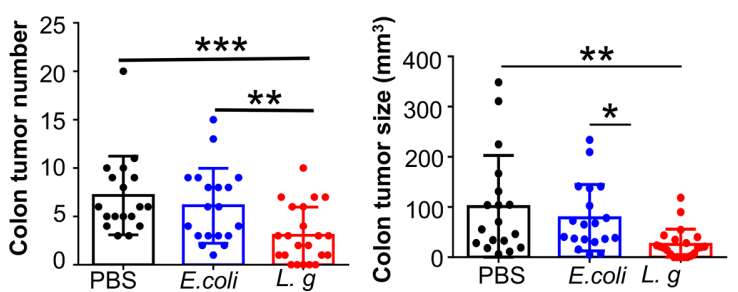

D

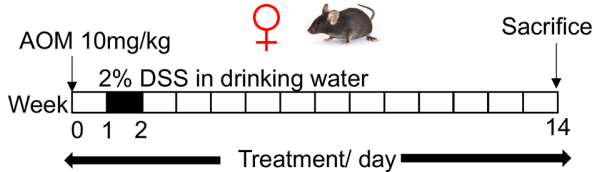

E

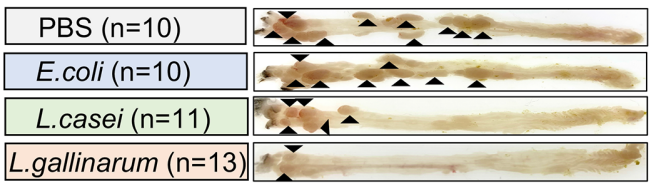

$\mathbf{F}$

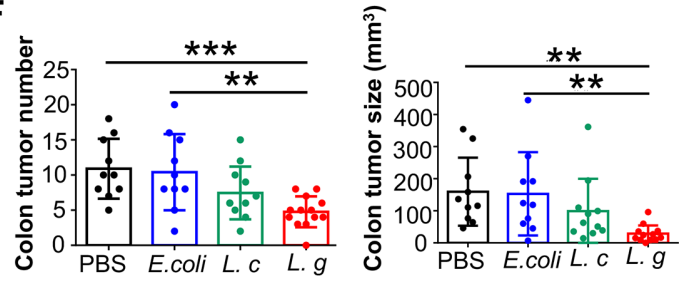

Figure 2 L. gallinarum protects against intestinal tumourigenesis in AOM/DSS-induced CRC mice. (A) Schematic diagram showing the experimental design, timeline of male AOM/DSS mouse model. (B) Representative images of colon tumour from male AOM/DSS mouse model. (C) Colon tumour number and tumour size in male AOM/DSS mice under different treatments. (D) Schematic diagram showing the experimental design, timeline of female AOM/DSS mouse model. (E) Representative images of colon tumour from female AOM/DSS mouse model. (F) Colon tumour number and tumour size in female AOM/DSS mice under different treatments. Each black triangle indicates one tumour location. P values are calculated by one-way analysis of variance. ${ }^{*} \mathrm{P}<0.05,{ }^{* *} \mathrm{p}<0.01,{ }^{* *} \mathrm{p}<0.001$. AOM, azoxymethane; DSS, dextran sulfate sodium; L. c, Lactobacillus casei; L. g, Lactobacillus gallinarum; PBS, phosphate-buffered saline; SI, small intestine.

E. coli strain MG1655 (1.0 $\times 10^{8}$ CFUs per mouse) as bacteria control, or PBS once daily for 8 weeks (figure 1A). During the period of gavage, there was no difference in body weight among groups (online supplemental figure 1A). The incidence of bloody stool in L. gallinarum group was lower than that of the PBS group $(\mathrm{p}<0.05)$ after gavage for 8 weeks (online supplemental figure 1B). Colonoscopy identified that colon tumour sizes in $L$. gallinarum group were visually smaller than the E. coli MG1655 or PBS groups (figure 1B). After sacrifice (figure 1C), significant reductions in tumour number $(E$. coli, $\mathrm{p}=0.034 ; \mathrm{PBS}, \mathrm{p}=0.005)$ (figure 1D) and tumour size (figure 1E) (E. coli, $\mathrm{p}<0.05$; PBS, $\mathrm{p}<0.05)$. L. gallinarum also significantly decreased tumour number (E. coli, p=0.003; PBS, $\mathrm{p}<0.05$ ) (figure 1D) and tumour size $(E$. coli, $\mathrm{p}=0.0012$; PBS, $\mathrm{p}<0.05)$ (figure $1 \mathrm{E}$ ) in the small intestine of $A p c^{\mathrm{Min} /+}$ mice (figure $1 \mathrm{~F}$ ).

To determine if the protective effect of $L$. gallinarum against CRC is gender-specific, we repeated the experiment in female $A p c^{\mathrm{Min} /+}$ mice (figure $1 G$ ). L. casei, a well-known LAB which has been reported to inhibit CRC progression through releasing small molecules, ${ }^{20}$ was used as a positive control. We observed consistent results as in the male $A p c^{\mathrm{Min} /+}$ mice, including decreased tumour development as captured by colonoscopy (figure $1 \mathrm{H}$ ) and tumour incidence in colon (figure 1I) and small intestine (figure 1J) in L. gallinarum-treated female $A p c^{\mathrm{Min} /+}$ mice. Significant reductions of both total tumour number (L. gallinarum vs E. coli, $\mathrm{p}<0.01 ;$ L. gallinarum vs PBS, $\mathrm{p}<0.01)$ and total tumour size (L. gallinarum vs E. coli, $\mathrm{p}<0.05$; L. gallinarum vs PBS, $\mathrm{p}<0.05)$ were also observed in L. gallinarum-treated female $A p c^{\mathrm{Min} /+}$ mice as compared with controls (figure $1 \mathrm{~K}$ and $\mathrm{L}$, online supplemental figure 2). Accordingly, L. casei slightly inhibited tumour formation in female $A p c^{\mathrm{Min} /+}$ mice (figure $1 \mathrm{~K}$ ), but its effect was not as strong as that of L. gallinarum. These results suggested that L. gallinarum abrogates intestinal tumourigenesis in both male and female $A p c^{\mathrm{Min} /+}$ mice.

\section{L. gallinarum protects against intestinal tumourigenesis in AOM/DSS-induced CRC mice}

To validate the tumor-suppressive effect of L. gallinarum on colorectal tumourigenesis, we established a colitis-associated CRC model, in which C57BL/6 mice aged 6 weeks were intraperitoneally injected with $10 \mathrm{mg} / \mathrm{kg}$ AOM, followed by $2 \%$ DSS administration for 1 week (figure 2A). L. gallinarum significantly reduced colorectal tumour number (E. coli, $\mathrm{p}<0.05$; PBS, $\mathrm{p}=0.007)$ and tumour size (E. coli, $\mathrm{p}=0.019$; PBS, $\mathrm{p}=0.0013)$ (figure 2B and C) in AOM/DSS-induced male CRC mice. Consistently, same results were observed in AOM/DSS-induced female CRC mice (figure 2D-F). During the period of gavage, there was no difference in body weight among different groups of mice (online supplemental figure 3 and online supplemental figure 4), indicating that L. gallinarum suppresses intestinal tumourigenesis in AOM/DSS-induced CRC in both male and female mice.

\section{L. gallinarum modulates the gut microbiota of $\mathrm{Apc}^{\mathrm{Min} /+}$ mice}

To investigate the effects of L. gallinarum on gut microbiota, we performed 16S rDNA gene sequencing on $A p c^{\mathrm{Min} /+}$ mouse stool samples after gavage of L. gallinarum for 8 weeks. The microbial abundance in L. gallinarum group significantly increased compared with PBS $(\mathrm{p}<0.05)$, while there was no difference between L. gallinarum and E. coli groups (figure 3A). Similarly, although the $\beta$-diversity of stool samples from L. gallinarumgavaged mice had a distinct trend compared with other two groups, there was no statistical significance (figure $3 \mathrm{~B}$ ). However, L. gallinarum could enhance abundances of some well-characterised commensal probiotics including Lactobacillus helveticus, Lactobacillus reuteri and OTUs from the Bacteroidetes phylum (figure $3 \mathrm{C}$ ). Moreover, some genera, such as Alistipes, Allobacullum, Dorea, Odoribacter, Parabacteroides and Ruminococcus with species of pathogenic potentials, exhibited significantly decreased abundances in mice treated with $L$. gallinarum compared with control groups (figure 3C). Taken 


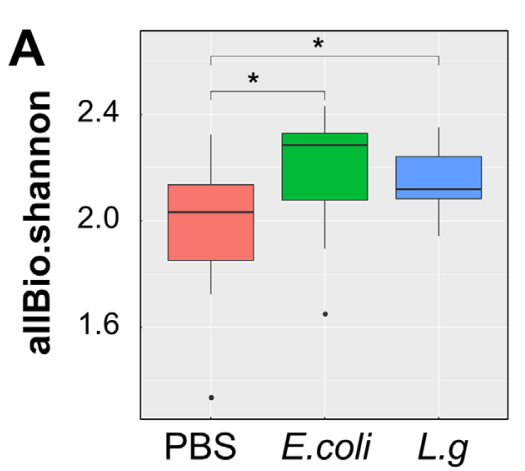

C
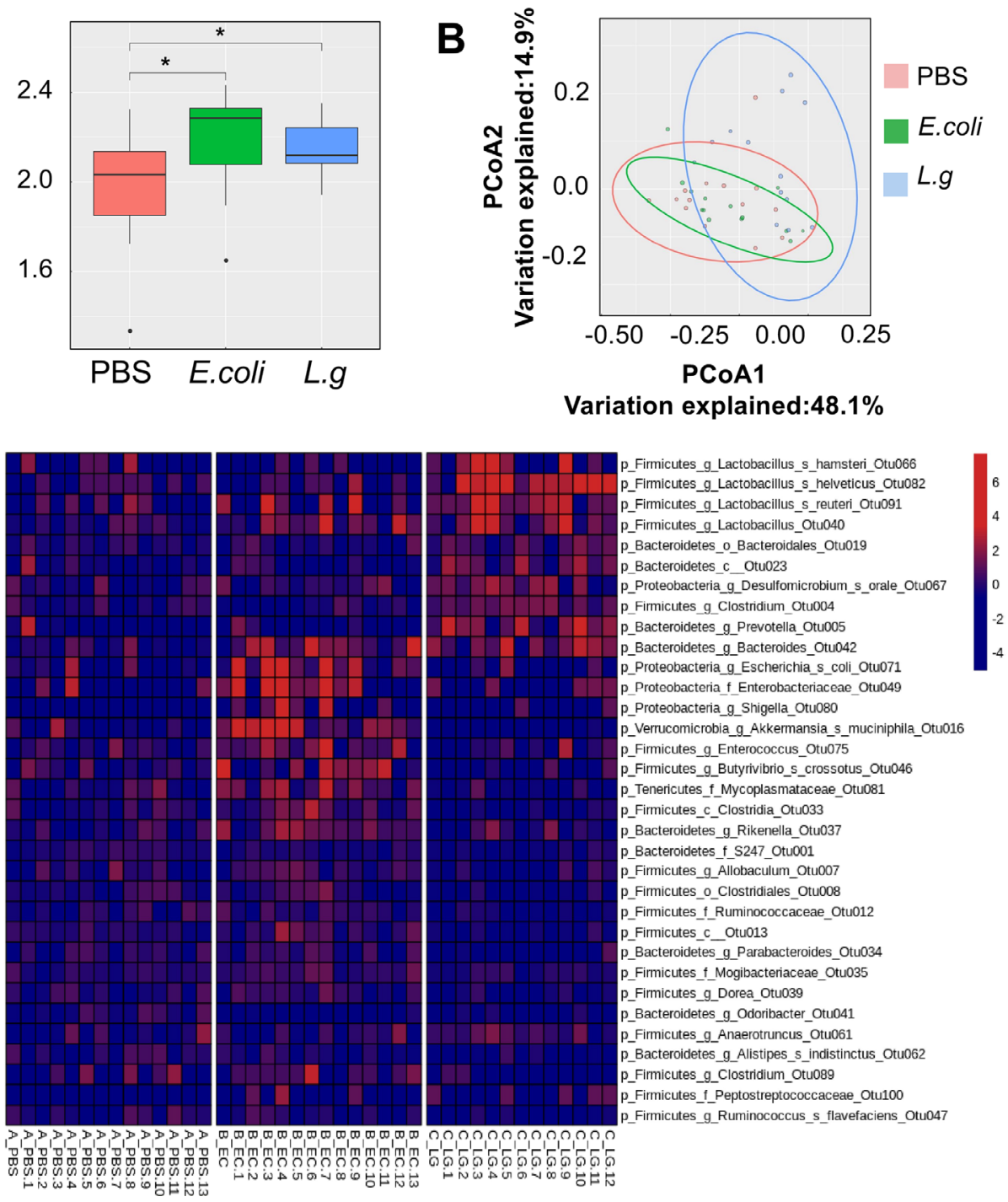

Figure 3 L. gallinarum modulates the gut microbiota of $A p \mathrm{C}^{\mathrm{Min} /+}$ mice. $16 \mathrm{~S} \mathrm{rDNA}$ gene analysis of intestinal microbiome. (A) Alpha-diversity analysis of luminal microbiota OTU at various taxonomic ranks. (B) Principal coordinate analysis (PCOA) of $\beta$-diversity based on Bray-Curtis dissimilarity matrix of OTU-level compositional profiles. Ellipses represent $95 \% \mathrm{Cls}$. Solid diamond-shaped points in black denote species scores, which were calculated using the vegan R-CRAN package. (C) Heatmap of differentially abundant bacterial OTUs using one-way analysis of variance, at p $<0.05$. L. g, L. gallinarum; OTU, operational taxonomic unit.

together, although L. gallinarum could not change the overall gut microbiota composition, it could enrich abundances of probiotics and potentially deplete gut pathogens.

\section{L. gallinarum supernatant inhibits the viability of colon cancer cells}

To validate the tumor-suppressive effect of $L$. gallinarum in vivo, we performed in vitro functional analyses using two CRC cell lines (HCT116 and LoVo) and a normal colonic epithelial cell line (NCM460) as control. Treatment with the culture supernatant of L. gallinarum significantly reduced the viability of CRC cell lines in a concentration-dependent manner, but not in normal colonic epithelial cell line as determined by cell viability assay (figure 4A-C). Similar results were observed in colony formation assay in which HCT116 (ECCS, $p=0.0002$; BHI, $p=0.0003$ ) and LoVo (ECCS, $p=0.0001$; BHI, $p=7.6 \times 10^{-5}$ ) showed significant decrease in colony compared with ECCS or BHI groups (figure 4D). These data indicated that the secreted molecules from L. gallinarum could suppress viability and colony-forming ability of CRC cells.

\section{L. gallinarum supernatant promotes apoptosis in CRC cells}

To determine the mechanism by which LGCS suppresses CRC cell viability, the effects of LGCS on apoptosis and cell cycle distribution were assessed quantitatively by flow cytometry with Annexin V-PE and 7-AAD staining. We found that LGCS significantly promoted apoptosis in CRC cell lines, HCT116 (ECCS, $\mathrm{p}=3.3 \times 10^{-6} ; \mathrm{BHI}, \mathrm{p}=5.1 \times 10^{-6}$ ) (figure $5 \mathrm{~A}$ ) and LoVo (ECCS, $\mathrm{p}=1.4 \times 10^{-6} ; \mathrm{BHI}, \mathrm{p}=3.6 \times 10^{-6}$ ) (figure $5 \mathrm{~B}$ ), while it had no effect on normal epithelial colonic cells, NCM460 (figure 5C). Moreover, we confirmed this apoptosisinducing property of LGCS on CRC organoids derived from 2 patients, 74 (ECCS, $\mathrm{p}=1.2 \times 10^{-5}$; BHI, $\mathrm{p}=0.0001$ ) and 816 (ECCS, $\mathrm{p}=0.0013$; BHI, $\mathrm{p}=0.0016$ ) (figure $5 \mathrm{D}$ and $\mathrm{E}$ ). By contrast, LGCS had no effect on cell cycle distribution in CRC cells (figure $5 \mathrm{~F}-\mathrm{H}$ ). 
A

HCT116 (5\%)

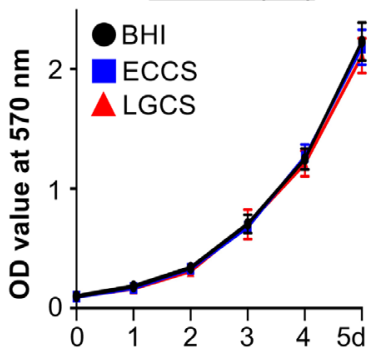

HCT116 (10\%)

HCT116 (20\%)
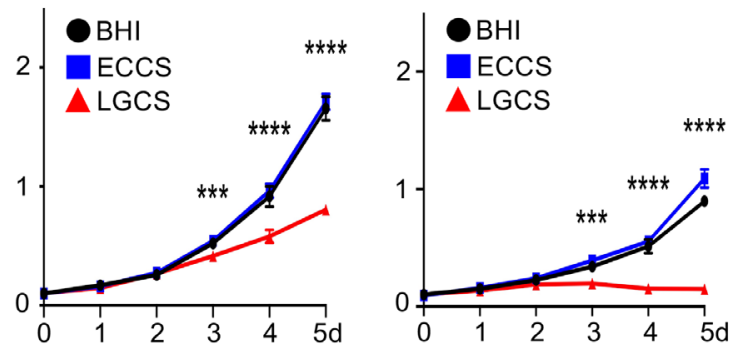

B

LoVo (5\%)

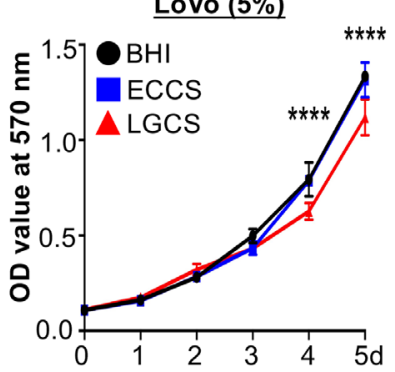

LoVo $(10 \%)$

LoVo $(20 \%)$
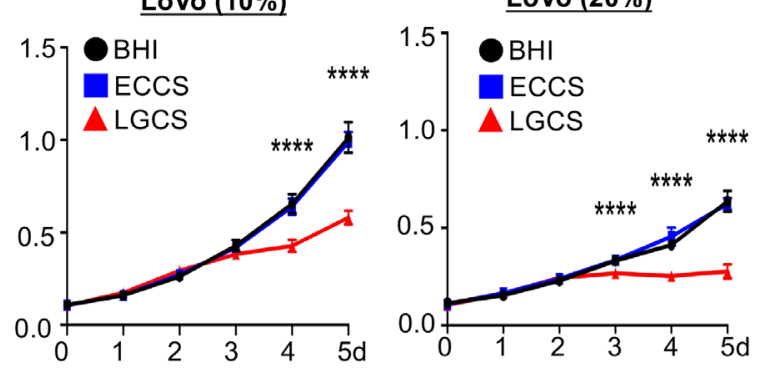

C

NCM460 (5\%)

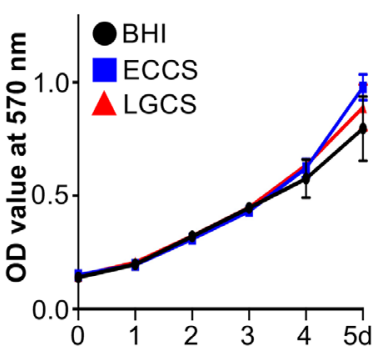

NCM460 (10\%)

NCM460 (20\%)
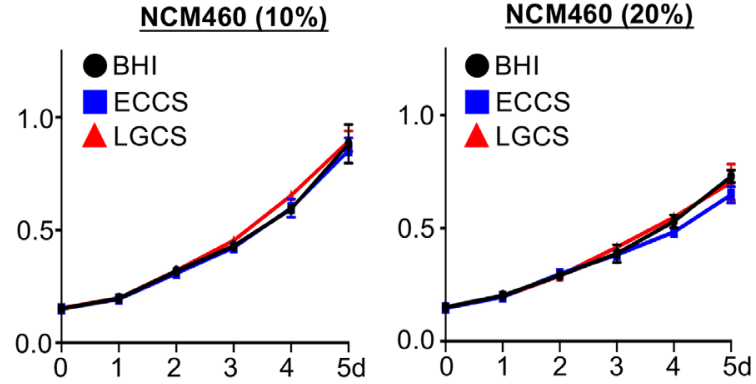

D
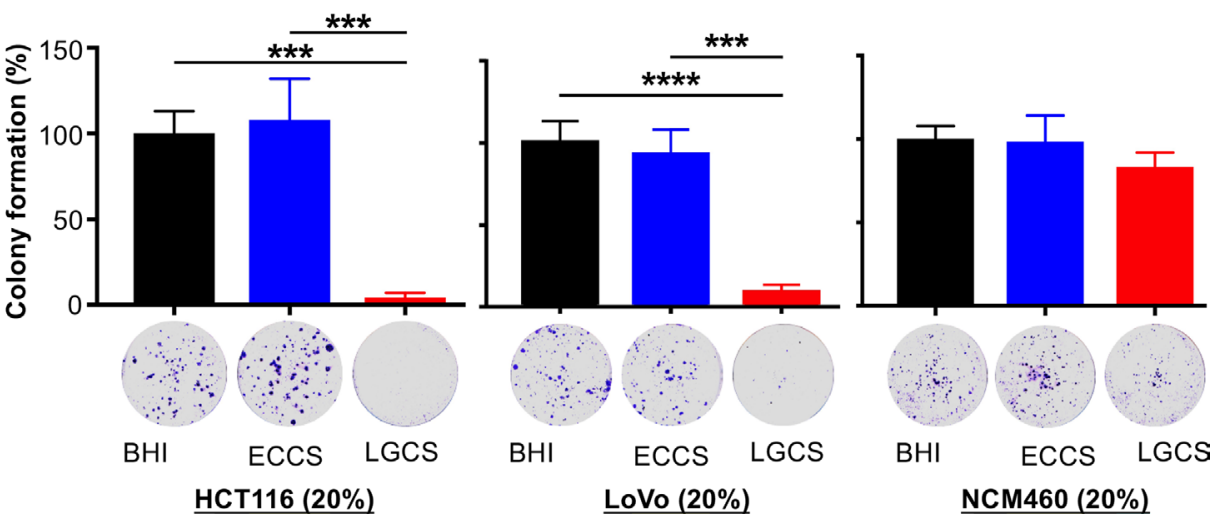

Figure 4 L. gallinarum supernatant inhibits the viability of colon cancer cells. The proliferation of cells was measured by 3-(4,5-dimethylthiazoly2-yl)-2,5-diphenyltetrazolium bromide assay. Different concentrations of culture supernatant were used for culturing CRC cell lines, HCT116 (5\%, $10 \%, 20 \%)$ and LoVo (5\%, 10\%, 20\%) and normal colonic epithelial cell line, NCM460 (5\%, 10\%, 20\%). (A) The culture supernatant of L. gallinarum, especially in $10 \%$ and $20 \%$, significantly suppressed the cell growth of HCT116 from day 4 to day 5 . (B) The cell growth of LoVo was also significantly suppressed by the culture supernatant of $L$. gallinarum with different concentrations; both $5 \%$ and $10 \%$ from day 4 to day 5 , and $20 \%$ from day 3 to day 5. (C) No change in cell growth could be observed in normal colonic epithelial cell line. (D) $20 \%$ LGCS suppressed colony formation of CRC cells. $\mathrm{P}$ values are calculated by two-way analysis of variance. ${ }^{* *} \mathrm{P}<0.001,{ }^{* * *} \mathrm{p}<0.0001$. BHI, brain heart infusion; CRC, colorectal cancer; ECCS, Escherichia coli culture supernatant; LGCS, Lactobacillus gallinarum culture supernatant.

Antitumour molecules produced from L. gallinarum are nonprotein with a molecular weight $<3 \mathrm{kDa}$

To investigate the features of anti-CRC molecules produced from L. gallinarum, bacterial culture supernatant was separated into low molecular weight (LMW) $(<3 \mathrm{kDa})$ and high molecular weight (HMW) (>3 kDa) fractions using $3 \mathrm{kDa}$ filter units. We found that decrease in viability of CRC cells was observed only in those treated with LGCS LMW fraction, while LGCS HMW fraction had no suppressive effect on CRC cells (figure 6A). Meanwhile, both heat-inactivated LGCS (figure 6B) and proteinase K-treated LGCS (figure 6C) retained the ability to reduce cell viability of CRC cells. Collectively, these results indicated that the anti-CRC properties of L. gallinarum could be induced by non-protein molecules with a molecular weight $<3 \mathrm{kDa}$. 
A
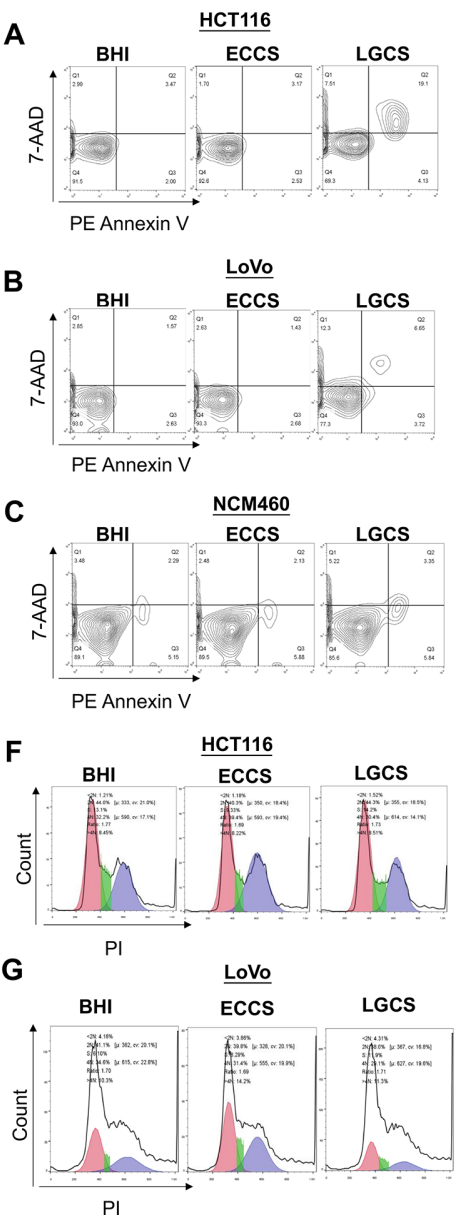
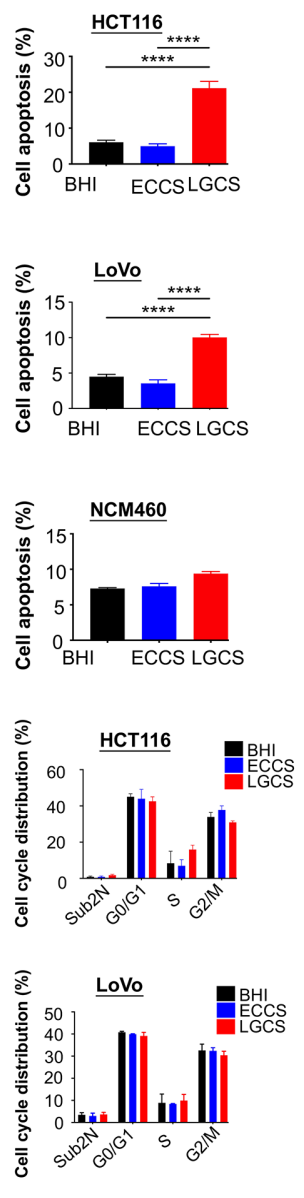

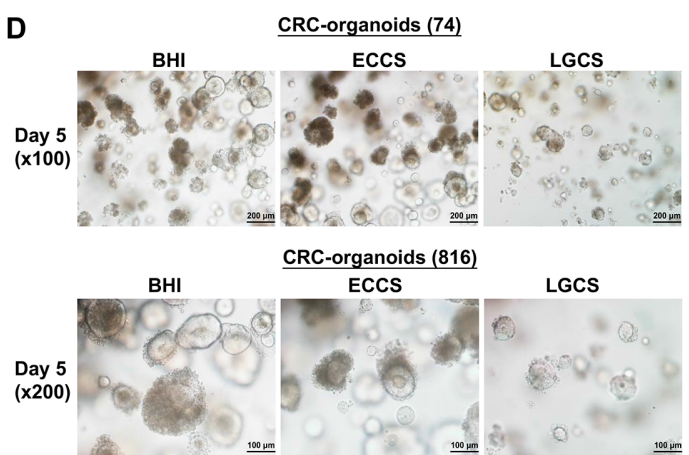

$\mathbf{E}$
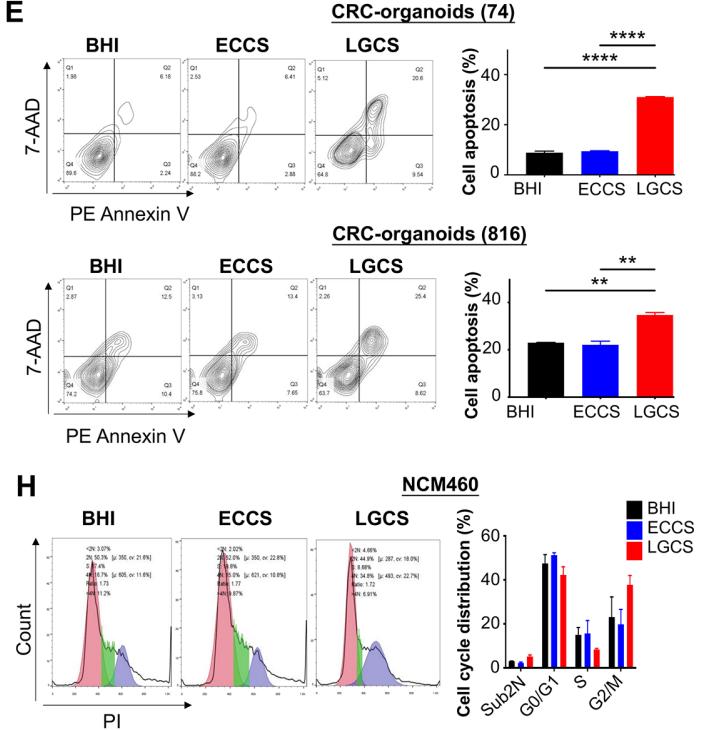

Figure 5 L. gallinarum supernatant promotes apoptosis instead of cell cycle arrest in CRC cells. (A) LGCS significantly promoted apoptosis including both early and late phases in two CRC cell lines HCT116, and (B) LoVo, but not in the normal colonic epithelial cell line (C) NCM460. (D) The size and number of CRC patient-derived organoids was visually reduced in medium containing $10 \%$ LGCS. (E) LGCS significantly promoted apoptosis including both early and late phases in CRC patient-derived organoids. (F) LGCS had no effect on cell cycle distribution in HCT116, (G) LoVo and (H) NCM460. P values are calculated by one-way analysis of variance. ${ }^{* *} \mathrm{P}<0.01,{ }^{* * * *} \mathrm{p}<0.0001$. BHI, brain heart infusion; CRC, colorectal cancer; ECCS, Escherichia coli culture supernatant; LGCS, Lactobacillus gallinarum culture supernatant; PI, propidium iodide.

We next performed non-targeted LC-MS/MS to identify anti-CRC metabolite(s) in the LGCS-LMW fraction (online supplemental table 2). Score plots of principal component analysis (PCA) showed clear separations among LMW fractions of LGCS, ECCS and BHI groups (figure 6D). Differential abundance analysis showed the critical products generated from L. gallinarum that may contribute to the anti-CRC effects (figure 6E). To verify if L. gallinarum-produced LMW molecules are responsible for the anti-CRC effect in vivo, we then examined the intestine metabolomics by using faecal samples from $A p c^{\mathrm{Min} /+}$ mice under different treatments (online supplemental table 3). Daily administration of L. gallinarum caused a significant overall compositional alteration of the gut metabolites as revealed by principal coordinate analysis (figure $6 \mathrm{~F}$ ). We found that metabolites including L-tryptophan, palmitic acid, 4-pyridoxic acid and gamma-L-glutamyl-L-glutamic acid were significantly upregulated in mice after L. gallinarum gavage as compared with control groups (figure 6G), which were also highly upregulated in LGCS (figure 6E). Among them, L-tryptophan was the most upregulated metabolite secreted by L. gallinarum. L-tryptophan is commonly converted into its downstream metabolites by gut microbiota. ${ }^{21}$ We anticipated that L. gallinarum-produced L-tryptophan and its downstream metabolites could be responsible for the anti-CRC effect exhibited by $L$. gallinarum.

\section{L. gallinarum produces and catabolises L-tryptophan to release indole-3-lactic acid to protect against CRC}

To further confirm L-tryptophan production by L. gallinarum and its conversion into downstream metabolites, we performed high-throughput targeted L-tryptophan metabolic profiling using culture supernatant at the late-stage stationary phase of $L$. gallinarum to ensure sufficient release and metabolic conversion of the metabolites (online supplemental table 4), as well as faecal samples from $A p c^{\mathrm{Min} /+}$ mice (online supplemental table 5). The PCA plot regarding L-tryptophan and its downstream metabolites showed obvious separations among LGCS, ECCS and BHI (figure 7A). Stool samples from L. gallinarum-treated $A p c^{\mathrm{Min} /+}$ mice also displayed clear separations from E. coli-treated or PBS mice (figure 7B). Differential abundance analysis showed that, in contrast to the log-phase culture supernatant, L-tryptophan was decreased in the stationary-phase LGCS (figure 7C), suggesting that L. gallinarum could catabolise L-tryptophan. In particular, we found ILA, which is one of the downstream catabolites from L-tryptophan, was highly enriched in both LGCS (figure 7C) and stool samples from L. gallinarum-tretaed $A p c^{\mathrm{Min} /+}$ mice (figure 7D).

We then performed functional investigation of ILA in CRC cell growth in vitro and in CRC tumourigenesis in vivo. As shown in 


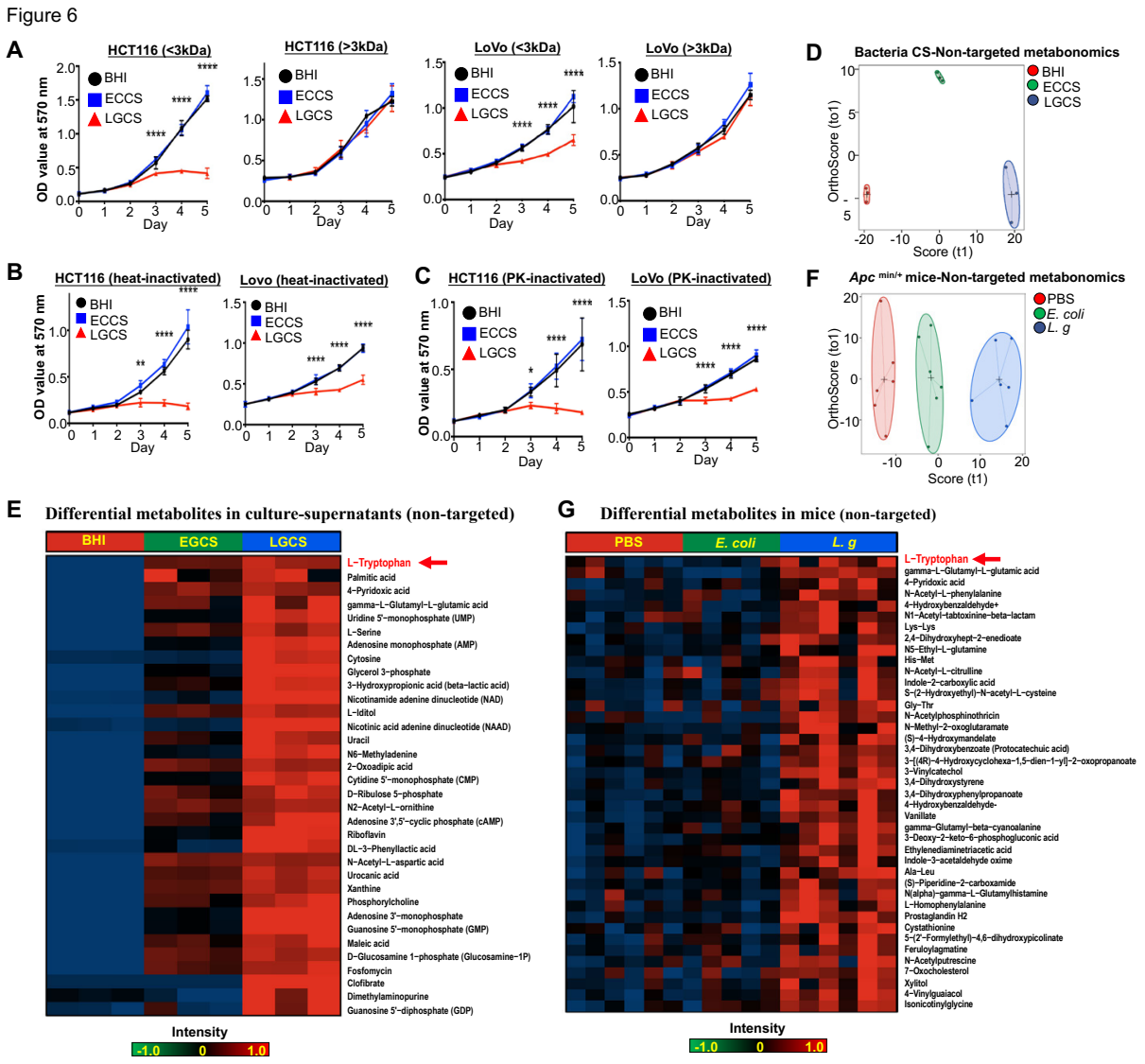

Figure 6 Antitumour molecules produced from L. gallinarum are non-protein with a molecular weight $<3 \mathrm{kDa}$. (A) LMW-LGCS but not HMWLGCS significantly suppressed cell growth of HCT116 and LoVo. (B) Decrease in proliferation of CRC cells was observed in heat-inactivated LGCS. (C) Decrease in proliferation of CRC cells was observed in PK-inactivated LGCS. (D) Score plots of PCA revealed clear separations of metabolites in culture supernatant of $L$. gallinarum, E. coli MG1655 and BHI groups. (E) Heatmap analysis revealed the abundance of different metabolites in LGCS, ECCS and BHI groups. (F) Score plots of PCA revealed clear separations among L. gallinarum, E. coli MG1655 and PBS-treated Apc ${ }^{\mathrm{Min} /+}$ mice. (G) Heatmap analysis revealed the abundance of different metabolites in the gut of $A p c^{\mathrm{Min} /+}$ mice under different treatments. $\mathrm{P}$ values are calculated by two-way analysis of variance or Student's t-test as appropriate. ${ }^{*} \mathrm{P}<0.05,{ }^{* *} \mathrm{p}<0.01,{ }^{* * * *} \mathrm{p}<0.0001$. BHI, brain heart infusion; CRC, colorectal cancer; ECCS, Escherichia coli culture supernatant; HMW, high molecular weight; LGCS, Lactobacillus gallinarum culture supernatant; LMW, low molecular weight; PBS, phosphate-buffered saline; PCA, principal component analysis; PK, proteinase K.

figure 7E, cell viability of CRC cells (HCT116 and LoVo) but not normal colonic epithelial cell NCM460 was substantially reduced when exposing to ILA at a concentration comparable to that of LGCS. Consistent with the effect of LGCS, ILA-treated CRC cells showed an increase in apoptosis (figure 7F). For in vivo experiments, gavage of ILA $(20 \mathrm{mg} / \mathrm{kg})$ into $A p c^{\mathrm{Min} /+}$ mice (figure $7 \mathrm{G}$ ) significantly reduced tumour number (figure $7 \mathrm{H}$ ) and tumour size (figure 7I). The amount of cells with positive TUNEL staining was also increased in ILA-treated tumour tissues, but not in adjacent normal tissues (figure $7 \mathrm{~J}$ ). Moreover, we have performed additional experiments to investigate if antagonising ILA could abrogate L. gallinarum medicated anticancer effect. As shown in online supplemental figure 6A, blocking ILA receptor, AhR, using AhR antagonist $(\mathrm{CH}-223191)^{22} 23$ abolished the effect of L. gallinarum in suppressing CRC in vitro (online supplemental figure $6 \mathrm{~B}$ ) and in $A p c^{\mathrm{Min} /+}$ mice (online supplemental figure $6 \mathrm{C}-6 \mathrm{H})$. Collectively, these data suggested that $L$. gallinarum could produce L-tryptophan, and at the same time, convert it to its downstream ILA and other metabolites. Thus, ILA could contribute, at least in part, to the tumor-suppressive effect of L. gallinarum .

\section{DISCUSSION}

In this study, we demonstrated for the first time that oral administration of $L$. gallinarum reduced intestinal tumour number and size in $A p c^{\mathrm{Min} /+}$ mice and confirmed in AOM/DSS-induced CRC mouse model, indicating that L. gallinarum suppresses CRC tumourigenesis in both male and female mice. From in vitro experiments, we found that small non-protein metabolites produced by L. gallinarum suppressed the growth of CRC cells and CRC patient-derived organoids by promoting apoptosis.

Certain probiotics can suppress the progression of CRC in preclinical experiments. ${ }^{24-29}$ For example, both living and heat-killed L. rhamnosus GG (LGG) have anti-CRC effect by promoting apoptosis in human CRC cells. ${ }^{30}$ In an animal model, LGG could suppress CRC development by increasing expressions of various pro-apoptotic proteins such as Bax, casp3 and p53. ${ }^{31}$ Several studies have suggested that regular consumption of probiotics may improve the imbalanced intestinal microbiota, thus reducing the chance of chronic inflammation and production of carcinogenic compounds during intestinal dysbiosis. ${ }^{32-34}$

In the present study, we found that L. gallinarum significantly enriched the abundance of well-characterised commensal probiotics, such as $L$. helveticus and L. reuteri. While some 
A

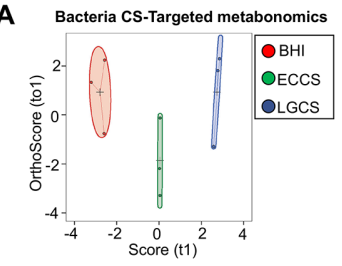

B
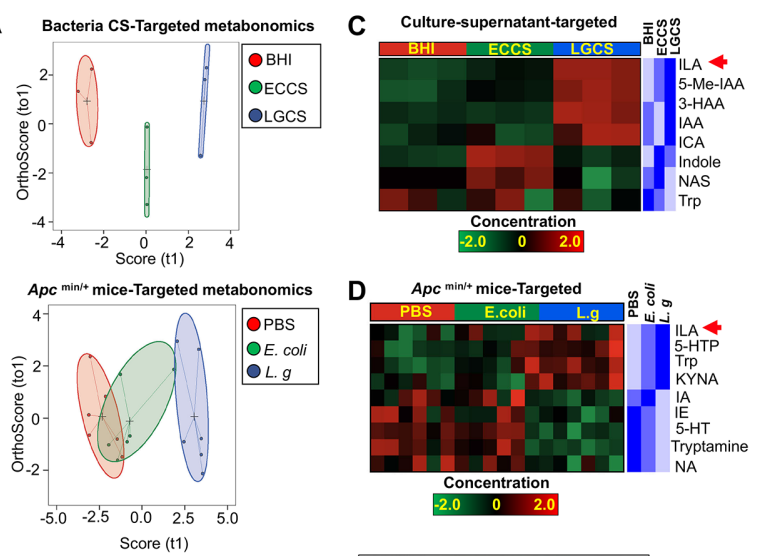

G
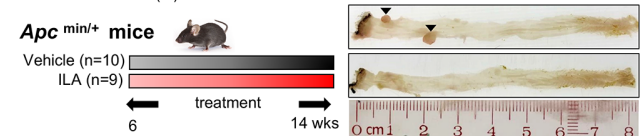

H
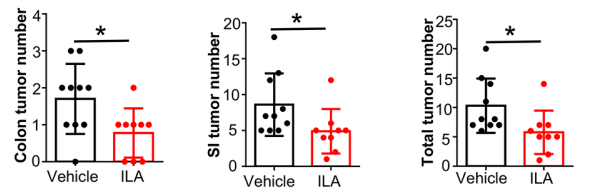

I
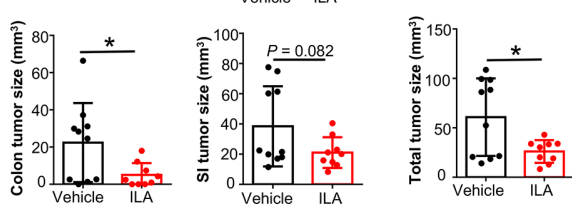

E
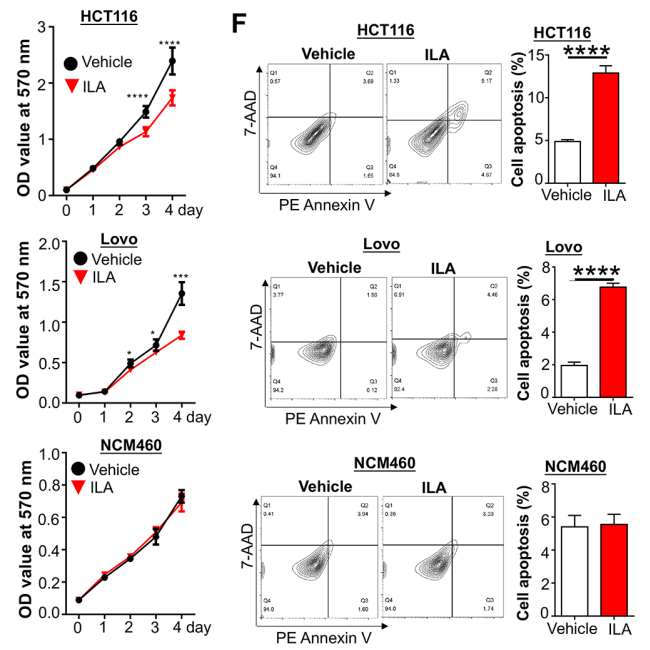

$J$

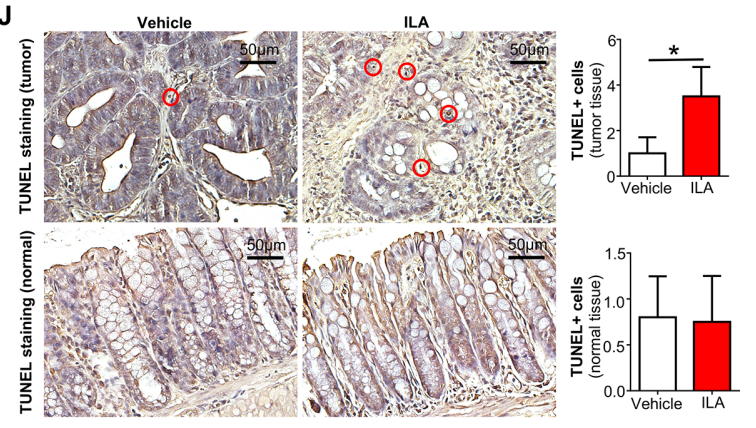

Figure 7 L. gallinarum produces and catabolises L-tryptophan to release ILA to protect against CRC. Targeted metabonomics on L-tryphtophan were performed on different culture supernatants and faecal samples from $A p \mathrm{C}^{\mathrm{Min} /+}$ mice under different treatments. (A) Score plots of PCA revealed clear separations among culture supernatant of L. gallinarum, E. coli MG1655 and BHI groups. (B) Score plots of PCA revealed clear separations among faecal samples from L. gallinarum-treated, E. coli MG1655-treated and PBS Apc ${ }^{\text {Min/t+ }}$ mice. (C) Heatmap analysis revealed the abundance of different metabolites in LGCS, ECCS and BHI groups. (D) Heatmap analysis revealed the abundance of different metabolites in the gut of $\mathrm{ApC}^{\mathrm{Min} /+}$ mice under different treatments. (E) The cell growth of CRC cells was significantly suppressed by ILA. (F) The cell apoptosis of CRC cells was significantly increased by ILA. (G) Schematic diagram showing the experimental design, timeline and representative macroscopic images of colons from of ILAtreated $\mathrm{Apc}^{\mathrm{Min} /+}$ mouse model. (H) Colon, small intestinal and total tumour number (colon+small intestinal) in $\mathrm{Apc}^{\mathrm{Min} /+}$ mice with or without ILA treatment. (I) Colon, small intestinal with or without ILA treatment. (J) TUNEL-positive staining cells in colon tissues of $A p c^{\mathrm{Min} /+}$ mice with or without ILA treatment. Each black triangle indicates one tumour location. P values are calculated by two-way analysis of variance or Student's $\mathrm{t}$-test as appropriate. ${ }^{*} \mathrm{P}<0.05,{ }^{* *} \mathrm{p}<0.01,{ }^{* * * *} \mathrm{p}<0.0001$. BHI, brain heart infusion; CRC, colorectal cancer; ECCS, Escherichia coli culture supernatant; ILA, indole-3-lactic acid; LGCS, Lactobacillus gallinarum culture supernatant; PBS, phosphate-buffered saline; PCA, principal component analysis.

potential pathogenic species, such as Alistipes, Allobacullum, Dorea, Odoribacter, Parabacteroides and Ruminococcus, ${ }^{35-37}$ were significantly depleted in mice treated with L. gallinarum, L. reuteri is known to suppress inflammation-associated colon carcinogenesis by producing histamine. ${ }^{38}$ Thus, L. gallinarum suppresses $\mathrm{CRC}$ at least in part through enriching abundances of probiotics and depleting potential CRC pathogens. Gut microbiota plays a critical role in CRC tumourigenesis. Our previous study showed that transplantation of faeces from patients with $\mathrm{CRC}$ can promote tumourigenesis in germ-free mice and AOMtreated mice. ${ }^{39}$ Another study demonstrated that transplantation of faecal samples from AOM/DSS mice to germ-free mice led to increased tumour development compared with those harbouring faecal samples from naïve healthy mice. ${ }^{40}$ These studies suggest that gut dysbiosis contributes to tumour susceptibility and alteration of the intestinal microbiota is an important determinant of colon tumourigenesis. Meanwhile, some studies found that probiotics can alter the composition of microbiota to alleviate cancer progression. For example, Lactobacillus salivarius Ren could suppress CRC tumourigenesis via modulating intestinal microbiota. ${ }^{41}{ }^{42}$ These findings collectively inferred that probiotics like L. gallinarum suppress CRC development through modulating gut microbial composition.

We also demonstrated that metabolites produced by L. gallinarum could suppress CRC cell viability through inducing apoptosis. Using metabolomic analysis, we found that L. gallinarum could produce L-tryptophan and convert L-tryptophan to its catabolites. We further identified that ILA, one of the L-tryptophan catabolites, was significantly increased in both LGCS and faecal samples of L. gallinarum-treated $A p c^{\mathrm{Min} /+}$ mice. ILA inhibited CRC cell viability in vitro and suppressed intestinal tumour development in vivo. A recent report suggested that L-tryptophan catabolites generated by the gut microbiota are important contributors in maintaining intestinal homeostasis. ${ }^{21}$ ILA was identified as a metabolite of breastmilk tryptophan, secreted by the probiotic Bifidobacterium longum to prevent inflammation. ${ }^{23}$ Production of ILA from gut microbes was reported to alleviate colitis in mice through inhibiting epithelial autophagy. ${ }^{43}$ ILA was also found to have regulatory effect on intestinal innate immunity, which plays a specific role in host-microbe crosstalk. CRC is influenced by the balance between microbial production of health-promoting metabolites (eg, short-chain fatty acids) and 
potentially carcinogenic metabolites (eg, secondary bile acids) ${ }^{44}$ Previous studies demonstrated the anticarcinogenic attributes of probiotic metabolites, especially for those produced by LAB. ${ }^{45}{ }^{46}$ For example, metabolites produced by Lactobacillus plantarum exhibited selective cytotoxicity via provoking antiproliferative activity and inducing apoptosis against malignant cancer cells. ${ }^{47}$ Ferrichrome derived from L. casei has a strong tumour-suppressive effect on CRC cells by inhibiting the JNK signalling pathway. ${ }^{20}$ Interestingly, we also observed stronger tumor-suppressive effect of LGCS as compared with LCCS (online supplemental figure 5). Thus, the anti-CRC feature of $L$. gallinarum could be also at least in part attributed to its released protective metabolites. However, whether ILA secreted from $L$. gallinarum is the main metabolite for CRC suppression requires further investigation.

In conclusion, to our understanding, this is the first study to demonstrate the anti-CRC effect of L. gallinarum. L. gallinarum protects against intestinal tumourigenesis. Such action is associated with modulation of the gut microbial composition and secretion of protective metabolites including ILA to promote apoptosis of cancer cells. These findings may facilitate the development of therapeutic strategy using probiotics for prevention of CRC.

\section{Author affiliations}

${ }^{1}$ Institute of Digestive Disease and Department of Medicine and Therapeutics, State Key Laboratory of Digestive Disease, Li Ka Shing Institute of Health Sciences, CUHKShenzhen Research Institute, The Chinese University of Hong Kong, Hong Kong, China

${ }^{2}$ Department of Gastroenterology, Osaka City University Graduate School of Medicine, Osaka, Japan

${ }^{3}$ Department of Anaesthesia and Intensive Care and Peter Hung Pain Research Institute, The Chinese University of Hong Kong, Hong Kong, China

${ }^{4}$ Institute of Precision Medicine, the First Affiliated Hospital, Sun Yat-sen University, Guangzhou, China

${ }^{5}$ Department of Immunology and Infectious Diseases/Genetics and Complex Disease, Harvard T. H. Chan School of Public Health, Boston, Massachusetts, USA

Contributors NS and QL were involved in study design, conducted the experiments and drafted the paper; ESHC, HCHL, WF, CL and ACYS performed the experiments; WXL, GN and OOC performed bioinformatics analyses; WKKW commented and revised the manuscript; FKLC supervised NS and commented on the study; JY designed, supervised the study and revised the manuscript.

Funding This study was supported by National Key R\&D Programme of China (No. 2020YFA0509200/2020YFA0509203), RGC Theme-based Res Scheme Hong Kong (T21-705/20-N), Lim Peng Suan Charitable Trust Research Grant Hong Kong, RGCCRF Hong Kong (C4039-19GF, C7065-18GF), RGC-GRF Hong Kong (14163817), Research Impact Fund Hong Kong (R4017-18F), Vice-Chancellor's Discretionary Fund Chinese University of Hong Kong.

Competing interests None declared.

Patient consent for publication Not applicable.

Ethics approval This study does not involve human participants. All animal studies were performed in accordance with guidelines approved by the Animal Experimentation Ethics Committee of The Chinese University of Hong Kong.

Provenance and peer review Not commissioned; externally peer reviewed.

Data availability statement All data relevant to the study are included in the article or uploaded as supplementary information.

Supplemental material This content has been supplied by the author(s). It has not been vetted by BMJ Publishing Group Limited (BMJ) and may not have been peer-reviewed. Any opinions or recommendations discussed are solely those of the author(s) and are not endorsed by BMJ. BMJ disclaims all liability and responsibility arising from any reliance placed on the content. Where the content includes any translated material, BMJ does not warrant the accuracy and reliability of the translations (including but not limited to local regulations, clinical guidelines, terminology, drug names and drug dosages), and is not responsible for any error and/or omissions arising from translation and adaptation or otherwise.

Open access This is an open access article distributed in accordance with the Creative Commons Attribution Non Commercial (CC BY-NC 4.0) license, which permits others to distribute, remix, adapt, build upon this work non-commercially, and license their derivative works on different terms, provided the original work is properly cited, appropriate credit is given, any changes made indicated, and the use is non-commercial. See: http://creativecommons.org/licenses/by-nc/4.0/.

\section{ORCID iDs}

Harry Cheuk Hay Lau http://orcid.org/0000-0003-3581-2909

William Ka Kei Wu http://orcid.org/0000-0002-5662-5240

Francis Ka Leung Chan http://orcid.org/0000-0001-7388-2436

Jun Yu http://orcid.org/0000-0001-5008-2153

\section{REFERENCES}

1 Siegel RL, Miller KD, Jemal A. Cancer statistics, 2019. CA Cancer J Clin 2019;69:7-34.

2 Rawla P, Sunkara T, Barsouk A. Epidemiology of colorectal cancer: incidence, mortality, survival, and risk factors. Prz Gastroenterol 2019;14:89-103.

3 Li Q, Hu W, Liu W-X, et al. Streptococcus thermophilus inhibits colorectal tumorigenesis through secreting $\beta$-galactosidase. Gastroenterology 2021;160:1179-93.

4 Mao J, Qi S, Cui Y, et al. Lactobacillus rhamnosus GG attenuates lipopolysaccharideinduced inflammation and barrier dysfunction by regulating MAPK/NF-KB signaling and modulating metabolome in the piglet intestine. J Nutr 2020;150:1313-23.

5 Lin R, Sun Y, Mu P, et al. Lactobacillus rhamnosus GG supplementation modulates the gut microbiota to promote butyrate production, protecting against deoxynivalenol exposure in nude mice. Biochem Pharmacol 2020;175:113868.

6 Soltan Dallal MM, Mojarrad M, Baghbani F, et al. Effects of probiotic Lactobacillus acidophilus and Lactobacillus casei on colorectal tumor cells activity (Caco-2). Arch Iran Med 2015;18:167-72.

7 Zhuo Q, Yu B, Zhou J, et al. Lysates of Lactobacillus acidophilus combined with CTLA4-blocking antibodies enhance antitumor immunity in a mouse colon cancer model. Sci Rep 2019:9:20128.

8 Salva S, Marranzino G, Villena J, et al. Probiotic Lactobacillus strains protect against myelosuppression and immunosuppression in cyclophosphamide-treated mice. Int Immunopharmacol 2014;22:209-21.

9 Shin R, Itoh Y, Kataoka M, et al. Anti-Tumor activity of heat-killed Lactobacillus plantarum BF-LP284 on Meth-A tumor cells in BALB/c mice. Int J Food Sci Nutr 2016:67:641-9.

10 Dai Z, Coker OO, Nakatsu G, et al. Multi-Cohort analysis of colorectal cancer metagenome identified altered bacteria across populations and universal bacterial markers. Microbiome 2018;6:70.

11 Moser AR, Pitot HC, Dove WF. A dominant mutation that predisposes to multiple intestinal neoplasia in the mouse. Science 1990;247:322-4.

12 Sápi J, Kovács L, Drexler DA, et al. Tumor volume estimation and quasi-continuous administration for most effective bevacizumab therapy. PLoS One 2015;10:e0142190.

13 Schloss PD, Westcott SL, Ryabin T, et al. Introducing mothur: open-source, platformindependent, community-supported software for describing and comparing microbial communities. Appl Environ Microbiol 2009;75:7537-41.

14 Needleman SB, Wunsch CD. A general method applicable to the search for similarities in the amino acid sequence of two proteins. J Mol Biol 1970;48:443-53.

15 Quast C, Pruesse E, Yilmaz P, et al. The Silva ribosomal RNA gene database project: improved data processing and web-based tools. Nucleic Acids Res 2013;41:D590-6.

16 DeSantis TZ, Hugenholtz P, Keller K, et al. NAST: a multiple sequence alignment server for comparative analysis of 16S rRNA genes. Nucleic Acids Res 2006;34:W394-9.

17 Edgar RC, Haas BJ, Clemente JC, et al. UCHIME improves sensitivity and speed of chimera detection. Bioinformatics 2011;27:2194-200.

18 Dembélé $D$, Kastner P. Fold change RANK ordering statistics: a new method for detecting differentially expressed genes. BMC Bioinformatics 2014;15:14.

19 Jin Y, Tang S, Li W, et al. Hemolytic E. coli promotes colonic tumorigenesis in females. Cancer Res 2016;76:2891-900.

20 Konishi H, Fujiya M, Tanaka H, et al. Probiotic-derived ferrichrome inhibits colon cancer progression via JNK-mediated apoptosis. Nat Commun 2016;7:12365.

21 Roager HM, Licht TR. Microbial tryptophan catabolites in health and disease. Nat Commun 2018;9:3294.

22 Wong CB, Tanaka A, Kuhara T, et al. Potential effects of Indole-3-Lactic acid, a metabolite of human bifidobacteria, on NGF-induced neurite outgrowth in PC12 cells. Microorganisms 2020;8. doi:10.3390/microorganisms8030398. [Epub ahead of print: $12032020]$

23 Meng D, Sommella E, Salviati E, et al. Indole-3-lactic acid, a metabolite of tryptophan, secreted by Bifidobacterium longum subspecies infantis is anti-inflammatory in the immature intestine. Pediatr Res 2020;88:209-17.

24 Jan G, Belzacq A-S, Haouzi D, et al. Propionibacteria induce apoptosis of colorectal carcinoma cells via short-chain fatty acids acting on mitochondria. Cell Death Differ 2002:9:179-88.

25 Tiptiri-Kourpeti A, Spyridopoulou K, Santarmaki V, et al. Lactobacillus casei exerts antiproliferative effects accompanied by apoptotic cell death and up-regulation of TRAIL in colon carcinoma cells. PLoS One 2016:11:e0147960.

26 Saxami G, Karapetsas A, Chondrou P, et al. Potentially probiotic Lactobacillus strains with anti-proliferative activity induce cytokine/chemokine production and neutrophil recruitment in mice. Benef Microbes 2017;8:615-23. 
27 Elfahri KR, Vasiljevic T, Yeager T, et al. Anti-colon cancer and antioxidant activities of bovine skim milk fermented by selected Lactobacillus helveticus strains. J Dairy Sci 2016;99:31-40.

28 El-Deeb NM, Yassin AM, Al-Madboly LA, et al. A novel purified Lactobacillus acidophilus 20079 exopolysaccharide, LA-EPS-20079, molecularly regulates both

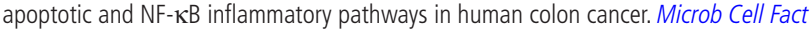
2018;17:29

29 Agah S, Alizadeh AM, Mosavi M, et al. More protection of Lactobacillus acidophilus than Bifidobacterium bifidum probiotics on azoxymethane-induced mouse colon cancer. Probiotics Antimicrob Proteins 2019;11:857-64.

30 Orlando A, Refolo MG, Messa C, et al. Antiproliferative and proapoptotic effects of viable or heat-killed Lactobacillus paracasei IMPC2.1 and Lactobacillus rhamnosus GG in HGC-27 gastric and DLD-1 colon cell lines. Nutr Cancer 2012;64:1103-11.

31 Gamallat Y, Meyiah A, Kuugbee ED, et al. Lactobacillus rhamnosus induced epithelial cell apoptosis, ameliorates inflammation and prevents colon cancer development in an animal model. Biomed Pharmacother 2016;83:536-41.

32 Hatakka K, Holma R, El-Nezami H, et al. The influence of Lactobacillus rhamnosus LC705 together with Propionibacterium freudenreichii ssp. shermanii JS on potentially carcinogenic bacterial activity in human colon. Int J Food Microbiol 2008; 128:406-10

33 Ohara T, Yoshino K, Kitajima M. Possibility of preventing colorectal carcinogenesis with probiotics. Hepatogastroenterology 2010;57:1411-5.

34 Liu Z, Qin H, Yang Z, et al. Randomised clinical trial: the effects of perioperative probiotic treatment on barrier function and post-operative infectious complications in colorectal cancer surgery - a double-blind study. Aliment Pharmacol Ther 2011:33:50-63.

35 Jeon H-J, Yeom Y, Kim Y-S, et al. Effect of vitamin C on azoxymethane (AOM)/dextran sulfate sodium (DSS)-induced colitis-associated early colon cancer in mice. Nutr Res Pract 2018;12:101-9.

36 Koh GY, Kane AV, Wu X, et al. Parabacteroides distasonis attenuates tumorigenesis, modulates inflammatory markers and promotes intestinal barrier integrity in azoxymethane-treated A/J mice. Carcinogenesis 2020;41:909-17.
37 Song $\mathrm{H}$, Wang $\mathrm{W}$, Shen $\mathrm{B}$, et al. Pretreatment with probiotic Bifico ameliorates colitis-associated cancer in mice: transcriptome and gut flora profiling. Cancer Sci 2018;109:666-77.

38 Gao C, Ganesh BP, Shi Z, et al. Gut Microbe-Mediated suppression of inflammationassociated colon carcinogenesis by luminal histamine production. Am J Pathol 2017; 187:2323-36.

39 Wong SH, Zhao L, Zhang X, et al. Gavage of Fecal Samples From Patients With Colorectal Cancer Promotes Intestinal Carcinogenesis in Germ-Free and Conventional Mice. Gastroenterology 2017;153:1621-33.

40 Zackular JP, Baxter NT, Iverson KD, et al. The gut microbiome modulates colon tumorigenesis. mBio 2013;4:e00692-13.

41 Zhang M, Fan X, Fang B, et al. Effects of Lactobacillus salivarius ren on cancer prevention and intestinal microbiota in 1, 2-dimethylhydrazine-induced rat model. $J$ Microbiol 2015;53:398-405.

42 Zhu J, Zhu C, Ge S, et al. Lactobacillus salivarius ren prevent the early colorectal carcinogenesis in 1, 2-dimethylhydrazine-induced rat model. J App/ Microbiol 2014;117:208-16.

43 Fan Q, Guan X, Hou Y, et al. Paeoniflorin modulates gut microbial production of indole-3-lactate and epithelial autophagy to alleviate colitis in mice. Phytomedicine 2020;79:153345.

44 Ou J, Carbonero F, Zoetendal EG, et al. Diet, microbiota, and microbial metabolites in colon cancer risk in rural Africans and African Americans. Am J Clin Nutr 2013;98:111-20

45 Lupton JR. Microbial degradation products influence colon cancer risk: the butyrate controversy. J Nutr 2004;134:479-82.

46 Putaala H, Mäkivuokko H, Tiihonen K, et al. Simulated colon fiber metabolome regulates genes involved in cell cycle, apoptosis, and energy metabolism in human colon cancer cells. Mol Cell Biochem 2011;357:235-45.

47 Chuah L-O, Foo HL, Loh TC, et al. Postbiotic metabolites produced by Lactobacillus plantarum strains exert selective cytotoxicity effects on cancer cells. BMC Complement Altern Med 2019;19:114. 